\title{
CREACIÓN E IMPLEMENTACIÓN DEL CENTRO UNADISTAS DE PENSAMIENTO PROSPECTIVO Y ESTRATÉGICO - CUPPE
}

\author{
Carlos William Mera Rodríguez, ${ }^{43}$ Marilú Avendaño Avendaño, \\ Sandra Patricia Quijano, César Augusto Naranjo, \\ Clara Isabel Vargas Espinosa y Diana Patricia Prado Vargas ${ }^{44}$
}

\section{RESUMEN}

El origen de este artículo es el proyecto de Investigación "Creación e implementación del centro de pensamiento prospectivo y estratégico - CUPPE", que busca implementar el centro Unadista de pensamiento prospectivo. Este documento se centrará en describir al CUPPE, como núcleo gestor del conocimiento especializado en Prospectiva Estratégica. A partir de allí, abordaremos cuatro aspectos importantes: La Prospectiva, La Gestión de Conocimiento, Los Centro de Gestión del Conocimiento Especializados (CGCE) y Las aplicaciones de la prospectiva. Para presentar esta propuesta el GIEPE (Grupo de Investigaciones y Estudios Prospectivos y Estratégicos) realizó un benchmarking a nivel nacional e internacional de los centros más importantes del mundo donde se analizaron sus estructuras, características y aplicaciones. Las conclusiones nos permiten confirmar que las universidades de alta calidad deben pensar seriamente en construir su futuro, que una herramienta poderosa y eficiente es la prospectiva y que el mejor dispositivo para este fin es la creación de Centros de Gestión del Conocimiento Especializados, en este caso, la creación e implementación del CuPPE, que permite congregar grupos, redes, investigadores, consultores, expertos nacionales e internacionales y organizaciones de prospectiva pensando en un mejor futuro. El Centro pretende desarrollar la gestión del conocimiento en las nuevas tecnologías articulando el medio académico-científico-productivo-estado, mediante la investigación, el desarrollo regional, la proyección comunitaria y la formación de una actitud mental proactiva y prospectiva que permita incrementar la competitividad y productividad del país.

Palabras clave: futuro, prospectiva, estrategia, gestión del conocimiento, centro de gestión de conocimiento especializado.

\section{ABSTRACT}

The origin of this paper is the research project "Creation and implementation of center prospective and strategic thinking - CUPPE," which in its first phase gave the following objective: Create and Implement UNAD Center Prospective and thinking Strategic at the National University Open and Distance (UNAD). This paper will focus on describing the Cuppe, manager at the core expertise in Strategic Foresight. Discussing the topic will address four major

43 Magister en Educación de la Pontificia Universidad Javeriana. Director del Grupo de Investigaciones y Estudios Prospectivos y Estratégicos - (GIEPE). Director del Nodo UNAD - Colombia de la Red de Escenarios y Estrategias en América Latina. Miembro de la World Futures Studies Federation - (WFSF). Universidad Nacional Abierta y a Distancia (UNAD). Carlos.mera@unad.edu. co.

44 Facultad de Ciencias Administrativas, Contables, Económicas y de Negocios, ECACEN, UNAD 
aspects: Foresight, Knowledge Management, The Center for Knowledge Management Specialist (CGCE) and prospective applications. To submit the present proposal GIEPE conducted a benchmarking national and international center in the world where they analyzed their structures, properties and applications. The findings allow us to confirm that high-quality universities should think seriously about their future and build a powerful and efficient the foresight and the best device for this purpose is the creation of a Knowledge Management Specialist center and in this case the creation and implementation of Cuppe, for assembling groups, networks, researchers, consultants, national and international experts and organizations of prospective thinking a better future. The Centre aims to develop knowledge management in new technologies articulating the academic-scientific-productive - the state through research, regional development, community outreach and mental development of a proactive and forward-looking attitude so as to increase competitiveness and productivity the country.

Key words: Future, Foresight, Strategy, Knowledge Management, Management Center of Expertise.

\section{INTRODUCCIÓN}

La prospectiva consiste según la OCDE (Organización para la Cooperación y el Desarrollo Económico (OCDE), 2000, en «tentativas sistemáticas para observar a largo plazo el futuro de la ciencia, la tecnología, la economía y la sociedad, con el propósito de identificar las tecnologías emergentes que probablemente produzcan los mayores beneficios económicos y sociales».

Es por esta razón que la Creación e Implementación del Centro Unadista de Pensamiento Prospectivo y Estratégico en la Universidad Nacional Abierta y a Distancia es un proyecto de vital importancia no sólo para la Universidad sino para el país, ya que nos permitiría contribuir como núcleo gestor de conocimiento especializado en prospectiva estratégica, a la solución de las diferentes problemáticas locales, regionales, nacionales e internacionales en los ámbitos político, económico, social, educativo, organizacional, territorial, tecnológico etc., a través del desarrollo de ejercicios prospectivos in situ que nos orienten en la construcción de múltiples escenarios alternativos que brinden un mejor futuro.

De otra parte, se determinó analizar la viabilidad del Centro Unadista de Pensamiento Prospectivo y Estratégico a través de varias herramientas metodológicas. Para la descripción y el planteamiento del problema se utilizó la metodología del marco lógico y específicamente el árbol de problemas (Ver Anexo núm.1 y núm. 2) respectivamente. En lo relacionado con la metodología se utilizó el Método Delphi - Encuestas a Expertos (de 36 contestaron 25). La encuesta se diseñó en formato virtual y nos permitió concluir claramente la viabilidad de la propuesta.

De igual manera, se utilizó como herramienta estratégica el benchmarking para realizar el análisis de la competitividad del centro mediante la comparación de lo que hacemos y cómo lo hacemos, contra lo que hacen los mejores centros de prospectiva en el mundo y cómo lo hacen (ver Anexo núm. 3). 
La Creación e Implementación del Centro Unadista de Pensamiento Prospectivo y Estratégico beneficiará a la Comunidad Científica, satisfaciendo la necesidad de anticiparnos a los desafíos en un mundo cada vez más globalizado, brindando las herramientas prospectivas y estratégicas necesarias para la planeación y desarrollo de proyectos que mantengan a la sociedad colombiana a la vanguardia de los constantes cambios. Económicamente la propuesta es muy rentable, ya que la creación del CUPPE, generará oferta y demanda de investigaciones, consultorías, ejercicios prospectivos, estudios, análisis que, primero le darán un buen nombre a la Universidad y en segunda instancia, unos ingresos que le permitirán reinvertir en formación, movilidad, compra de mejores y actualizados software, etc. Académicamente, la propuesta beneficia a toda la comunidad académica ya que permite la formación y permanente actualización en temas relacionados con la prospectiva, además, se puede aprovechar la utilización de estas redes nacionales e internacionales en beneficio de la comunidad universitaria.

\section{OBJETIVOS}

Crear e implementar el centro Unadista de pensamiento prospectivo y estratégico (CUPPE), en la Universidad Nacional Abierta y a Distancia (UNAD), como núcleo gestor de conocimiento especializado en prospectiva estratégica.

\section{ANTECEDENTES}

\section{Prospectiva}

La prospectiva parte del concepto que el futuro aún no existe y "se puede concebir como un realizar múltiple" (Jouvenel, 1968) y que "depende solamente de la acción del hombre" (Godet, 1987). Por esa razón, el hombre puede construir el mejor futuro posible, para lo cual debe tomar las decisiones correctas en el momento apropiado.

Etimológicamente la palabra "prospectiva" se deriva del vocablo latino "prospectivus", que denota todo aquello relativo a la visión, al conocimiento, a la comprensión, a la mira. El significado más preciso lo da el verbo "prospicere", mirar a lo lejos, mirar más allá, obtener una visión de conjunto, a lo largo y a lo ancho.

Es importante resaltar que nuestra propuesta es la creación de un centro de pensamiento prospectivo y estratégico donde este última tenga un grado de importancia igual al de la prospectiva ya que la Estrategia según Mintzberg y Quinn (1993), es un "Patrón o plan que integra las principales metas y políticas de una organización, y, a la vez, establece la secuencia coherente de las acciones a realizar".

En el ámbito teórico, se destacan Gastón Berger y Bertrand y Hugues de Jouvenel como precursores de la prospectiva en Francia, a mediados de los años sesenta, y luego retomada por Michel Godet y en esta parte del mundo contamos con representantes como Francisco José Mójica, Eduardo Raúl Balbi, Tomas Miklos entre otros. 
Berger (1967), uno de los primeros inspiradores de la prospectiva en Francia, define la prospectiva como la ciencia que estudia el futuro para comprenderlo y poder influir en él. Jouvenel (1993), plantea que existen dos formas de ver el futuro; la primera como una realidad única, propia por los oráculos, profetas y adivinos. La segunda, como una realidad múltiple, estos son los futuros posibles que de Jouvenel denominó futuribles.

Godet (1993), la define como una «indisciplina intelectual» que toma la forma de una «reflexión para iluminar la acción del presente con la luz de los futuros posibles». Expresaba que la Prospectiva permitía realizar una lectura más aproximada de la realidad que la previsión, asimilando a esta última al "Forecasting". Las principales objeciones provienen del enfoque predictivo de la previsión en contraste con el planteamiento constructivo de la Prospectiva. La posición prospectiva está inmersa en la actitud proactiva, pues la probabilidad de que algo ocurra es directamente proporcional a la actividad que se despliega para que así sea.

Para Balbi (2008), la Prospectiva es la disciplina que ha conseguido convertirse en la herramienta clave de esa construcción del futuro deseado y posible, y en particular, sus últimos desarrollos que arriban a la llamada Prospectiva Estratégica constituyen el basamento fundamental del liderazgo y del management modernos.

Gil (2005), concibe la prospectiva de dos formas: como una "disciplina intelectual" o una "indisciplina intelectual" que toma la forma de una "reflexión para iluminar la acción del presente con la luz de los futuros posibles". En palabras del francés Godet (1993), esta disciplina propone analizar las posibles evoluciones de una organización o territorio - $\mathrm{O}$ parte de él- en un horizonte de tiempo determinado, teniendo en cuenta las interacciones que ésta tiene con sus entornos endógenos y exógenos para, de esta forma, elegir su mejor alternativa de futuro posible, a través de un ejercicio colectivo donde convergen todas las voluntades de los actores que tienen que ver con dicha organización o territorio, como una herramienta de construcción social del futuro que motiva y permite la generación de una cultura "anticipatoria".

\section{Gestión del conocimiento}

El centro de gestión del conocimiento especializado en prospectiva estratégica se fundamenta teórica y conceptualmente en la gestión del conocimiento de Nonaka y Takeuchi (1995), que tratan sobre el modelo de generación de conocimiento mediante dos espirales de contenido epistemológico y ontológico, constituida en una espiral permanente de transformación de conocimiento, que se desarrolla por medio de la socialización, exteriorización, combinación e interiorización para entender la naturaleza dinámica de la creación de éste. Para manejar tal modelo con eficacia tienen en cuenta 4 fases que se pueden apreciar en el siguiente gráfico: 
Figura 1. Proceso de conversión del conocimiento en la organización

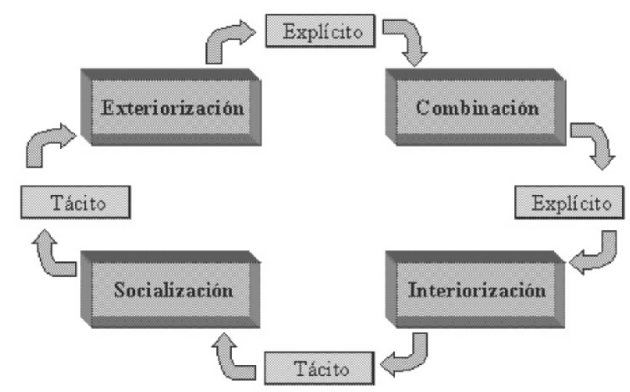

Fuente: NONAKA, I. y TAKEUCHI, H. 1995.

En suma, como el conocimiento es transferido hacia arriba y hacia abajo en la organización, la espiral de conocimiento se expande a través de diferentes modos de conversión, de tácito en explícito y viceversa. Para explicarlo mejor, Ikujiro Nonaka e Hirotaka Takeuchi proponen en 1995 un modelo de proceso de creación de conocimiento.

De igual manera, sustentamos nuestra propuesta teniendo en cuenta lo expresado por Drucker (2003), en el sentido de que, la esencia de la dirección es, cómo se puede aplicar de la mejor forma un conocimiento existente para poder crear otro conocimiento nuevo o reciclado.

Por otra parte, es importante resaltar la teoría de Senge (1992): quien en su libro La quinta disciplina sienta las bases de las denominadas organizaciones inteligentes. "Learning Organizations" que son "organizaciones donde la gente expande continuamente su aptitud para crear los resultados que desea, se cultivan nuevos y expansivos patrones de pensamiento prospectivo, la aspiración colectiva queda en libertad, y la gente continuamente aprende a aprender en conjunto" y en relación con los centros dice: "Las organizaciones que utilizan prácticas colectivas de aprendizaje — como centro de conocimiento- están bien preparadas para prosperar en el futuro, porque serán capaces de desarrollar cualquier habilidad que se requiera para triunfar".

\section{Centros de gestión de conocimiento especializado}

El centro de gestión del conocimiento es un sitio encargado de introducir procesos administrativos, productivos e innovadores de conocimiento, a través de la prestación de servicios de consultoría, evaluación y ejecución de proyectos a nivel nacional, teniendo como objetivo un desarrollo sostenible de carácter investigativo, científico y tecnológico.

El centro unadista de pensamiento prospectivo es el núcleo gestor que desarrolla y aplica modelos educativos, metodologías y estrategias para promover el conocimiento y brindar asesoría a entidades o instituciones en lo que a estudios de futuro se refiere. 
Según Senge, las organizaciones deben incorporar tres prácticas sistémicas en la gestión del conocimiento: mejorar continuamente procesos y productos, aprender a explotar el éxito y aprender a innovar, lo cual pretende realizar la UNAD, al crear e implementar el Centro Unadista de Pensamiento Prospectivo y Estratégico (CUPPE). Centro que se fundamentará en las corriente voluntarista de la prospectiva y determinista del FORESIGHT, utilizando software de LIPSOR sin dejar de lado el FORESIGHT, que parten principalmente de las técnicas y conocimientos de los maestros de la prospectiva Gastón Berger, Michel Godet y Francisco José Mojica, quienes coinciden en afirmar que el futuro está en nuestras manos y, por lo tanto, lo debemos construir.

Para realizar el análisis comparativo con otros centros se realizó un benchmarking a nivel mundial, la síntesis de este estudio se presenta en el siguiente cuadro (ver anexo núm. 3).

\section{Aplicaciones de la Prospectiva}

Balbi (2008), plantea las siguientes aplicaciones de la prospectiva. De manera muy breve y elemental referencia los distintos campos de aplicación directa de la prospectiva estratégica, podemos citar seguidamente nueve campos principales. Mera (2009), plantea dos campos adicionales.

Principales aplicaciones de la Prospectiva Estratégica:

a) Prospectiva política: se aplica al diseño de Políticas de Estado y de Gobierno. Políticas Públicas en general. Instrumentación y logro de la funcionalidad integral del sector público.

b) Prospectiva para la educación: políticas, contenidos, áreas, demandas, investigación, estructuras, modos y alcances de la educación.

c) Prospectiva territorial: se desarrolla en la organización de un territorio, sus recursos y su gente para el desarrollo sustentable, alta calidad de vida, cuidado del ambiente y logro de la seguridad humana.

d) Prospectiva urbana: se utiliza en las organizaciones de áreas urbanas, sus recursos, su gente y sus áreas satélites para el desarrollo sustentable, alta calidad de vida, cuidado del ambiente y logro de la seguridad humana (puede ser una parte integrante de la anterior).

e) Prospectiva científica, tecnológica y de innovación: diseño de políticas, estrategias y programas para lograr la I\&D en C\&T y las innovaciones necesarias para el desarrollo sustentable de regiones, ciudades y sectores.

f) Prospectiva organizacional: se encarga del estudio de futuro de las diferentes organizaciones y pretende darle respuesta a las preguntas: ¿Cuál deberá ser la organización, misión, tareas y competencias de mi organización en el año X?

g) Prospectiva competitiva: se aplica a la organización y puesta en marcha de sistemas integrados de producción, servicios, exportación, provisión de servicios especializados, negocios, etc. (clústeres, GACs, Consorcios)

h) Prospectiva de seguridad: se utiliza en el análisis de los riesgos y oportunidades en seguridad física, ambiental, industrial y del trabajo, frente a situaciones normales, excepcionales y de crisis. 
i) Prospectiva en salud: análisis y elaboración de políticas, contenidos, áreas, demandas, investigación, estructuras, modos, alcances de la salud

j) Prospectiva social, (Baena 2004): es la encarga de construir alternativas para la inclusión, seguridad humana (en todos los campos, alimentario, ambiental, de salud, comunitario, físico, político, económico).

k) Prospectiva comunitaria: (Ruiz 2000): promueve la construcción comunitaria del futuro; fortalecer la toma de decisiones, dis-minuir el costo social y mejorar la calidad humana en nuestro planeta.

\section{METODOLOGÍA DE FUTUROS}

Este proyecto se desarrolló metodológicamente a través de la investigación cualitativa dado que se estudia la prospectiva a nivel nacional e internacional y se hace una interpretación a partir de la descripción de otros centros y observatorios prospectivos para comprender su funcionamiento, características, estructura, portafolio de servicios, etc.

En esta investigación cualitativa no se descubre sino que se construye el conocimiento a través de la interacción de las fases heurística (investigación), hermenéutica (análisis interpretativo) y dialéctica (transformación a partir de la integración entre la teoría y la práctica). Los métodos de investigación que se utilizaron fueron la observación y el análisis del estado del arte de la prospectiva elaborado por el GIEPE.

En primer lugar, se hizo un estudio exploratorio, investigando sobre el comportamiento que ha tenido la prospectiva a nivel mundial, los centros y observatorios prospectivos existentes en los cinco continentes y en Colombia y la especialización de cada uno de ellos. Luego, se realizó un estudio descriptivo, identificando las características más relevantes y que de una u otra manera se podían implementar en el CUPPE.

Las técnicas utilizadas durante la investigación fueron la documental que se apoyó principalmente en la técnica de consulta bibliográfica, y generó un proceso de análisis y reflexión y la vertiente empírica o investigación de campo, que se basa en datos extraídos de experiencias exitosas de expertos prospectivistas a nivel mundial, a través de las técnicas de encuesta y entrevista y la vista a páginas web de instituciones públicas y privadas, organizaciones académicas y comerciales, para recoger información pertinente al comportamiento de Centros y Observatorios Prospectivos. El método delphi fue el seleccionado para la el desarrollo de esta investigación.

\section{Método Delphi - Encuesta a expertos}

Métodos de expertos: se basan en la consulta a personas que tienen grandes conocimientos sobre el entorno en el que la organización desarrolla su labor. Estas personas exponen sus ideas y finalmente se redacta un informe en el que se indican cuáles son, en su opinión, las posibles alternativas que se tendrán en el futuro. 


\section{Aplicación}

1. Contactar expertos

2. Enviar un cuestionario

3. Analizar las respuestas (áreas de acuerdo)

4. Enviar un análisis resumido de las respuestas

5. Repetir el proceso hasta que se estabilice

\section{Etapas:}

1. Preguntar a los expertos sus predicciones separadamente

2. Compilar las predicciones individuales

3. Informar a cada experto las predicciones de los otros

4. Revisar las predicciones a la luz de las otras opiniones

5. Repetir hasta lograr consenso con las otras opiniones

Figura 2. Proceso Delphi

\section{PROCESO DELPHI}

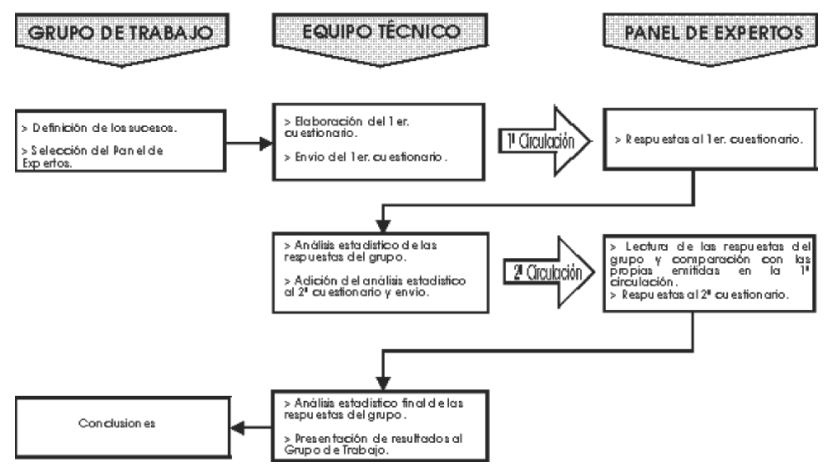

El formato de la encuesta (Ver anexo 3) se encuentra alojado en la dirección electrónica http://www.encuestafacil.com/RespWeb/QN.aspx?PE=r9FTmrqMZhk\%3d.

La encuesta fue aplicada a un grupo de 36 expertos de los cuales 25 la respondieron, entre estos contamos con la colaboración de expertos prospectivistas externos Nacionales e Internacionales y con un grupo de expertos internos que laboran en la Universidad. El listado completo de expertos es el siguiente:

Rector (1)

Vicerrectores (3)

Decanos de Escuelas (4)

Representante de los Coordinadores Académicos Nacionales (1) 
Representante de los Secretarios Académicos de Escuelas (1)

Representante de Directores de Nodo (1)

Representantes de Directores de CEAD (1)

Representante de Coordinadores Administrativos de Zona (1)

Representante de Coordinadores Académicos y de Investigación zonales (1)

Representante de Coordinadores de CERES (1)

Representante de Coordinadores de programa académico (1)

Representante del personal académico (3)

Representante de los Estudiantes (5)

Representante de los Egresados (2)

Representante del Sector Productivo (4)

Expertos prospectivistas (6)

\section{RESULTADOS PARCIALES}

Para sintetizar el análisis del instrumento se dividió la encuesta en cuatro grandes bloques, como aparece en el aplicativo (Ver anexo núm. 4) y los resultados fueron:

En el primer bloque de preguntas los expertos respondieron en un $92 \%$ de forma positiva para determinar la importancia de la prospectiva.

El segundo bloque que hace referencia a la importancia de la creación e implementación del Centro Unadista de Pensamiento Prospectivo los expertos respondieron en un $100 \%$ que la propuesta es pertinente y viable ya que fortalecería la cultura del pensamiento prospectivo en la UNAD y en el país.

Para el tercer bloque los expertos encuestados resaltan la importancia de la utilización de tecnologías de Información y Comunicación como herramientas flexibles y accesibles para la divulgación y diseño de estrategias prospectivas del cuPPE en un 96\%.

En el cuarto bloque dio como resultado que un $92 \%$ de los encuestados consideran que el CUPPE a su interior debe contar con una herramienta como el Observatorio de Prospectiva Estratégica que brinde información actualizada y precisa de los campos interdisciplinarios del saber y del conocimiento.

Es muy importante también resaltar que para los expertos el área de aplicación prospectiva de la Universidad es muy amplio y probablemente podría trabajar en todos los que se presentan en el documento ya que por ser una Universidad Nacional, con sedes en todo el país y que su naturaleza es social, comunitaria, la ubican en un multicontexto que requiere de respuestas específicas a sus múltiples problemáticas.

Por último, la investigación en el anexo núm. 6 presenta los resultados esperados en aspectos como Generación de nuevo conocimiento, Fortalecimiento de la Comunidad científica, Apropiación Social del Conocimiento, Impactos esperados. 


\section{PERSPECTIVAS DEL TRABAJO}

Es de vital importancia continuar con la construcción de la Plataforma Prospectiva de la UNAD, como herramienta de constante construcción del futuro, de forma ordenada y significativa. (Ver anexo núm. 5).

En la segunda fase del proyecto se pretende diseñar el direccionamiento estratégico, la estructura organizacional y el portafolicio de servicios del Centro Unadista de Pensamiento Prospectivo y Estratégico.

Con el apoyo de la Red de Gestión Prospectiva y Estratégica (Redefuturo) y la Red de Escenarios y Estrategias en América Latina de la cual la Unad fue designada como Nodo UNAD - Colombia, el Centro buscará construir alianzas, convenios y consocios con otras redes, centros y grupos de investigación que le permitan ser reconocido y visibilizar la gestión de conocimiento en el tema de la prospectiva a nivel nacional e internacional.

Prospectivamente se visualiza en el futuro un fuerte trabajo de asesoría y acompañamiento de los nuevos centros de gestión de conocimiento especializado de la Universidad en el tema de creación e implementación de unidades prospectivas al interior de estos.

\section{CONCLUSIONES}

La Investigación nos permitió confirmar que las Universidades de alta calidad deben pensar seriamente en construir su futuro, que una herramienta poderosa y eficiente es la prospectiva y que el mejor dispositivo para este fin es la creación de Centros de Gestión del Conocimiento Especializados y en este caso la creación e implementación del CUPPE.

La utilización de una herramienta estratégica como el benchmarking nos permitió identificar claramente las características, campos y aéreas de conocimiento y los mercados objetivos que tiene cada uno de los centros de prospectiva del mundo, lo cual nos permite determinar claramente en qué campos de aplicación y a qué mercado objetivo debemos dirigir el centro de pensamiento.

Por último, como dice Peter Senge: "Las organizaciones que utilizan prácticas colectivas de aprendizaje - como centro de conocimiento - están bien preparadas para prosperar en el futuro, porque serán capaces de desarrollar cualquier habilidad que se requiera para triunfar". Con esta idea se sustenta claramente que la Creación e Implementación del Centro Unadista de Pensamiento Prospectivo y Estratégico CUPPE, es un proyecto de vital importancia para la Universidad y el País. 


\section{REFERENCIAS BIBLIOGRÁFICAS}

BAENA, Guillermina. (2007), Prospectiva Política guía para su comprensión y práctica. México: Universidad Nacional Autonoma de México y Convenio Andrés Bello.

BALBI, Eduardo. (2008), Metodología de la Investigación de futuros. Argentina: CelGyP y The Millenum Project.

BERGER. Gastón. (1957), "Sciences humaines et prévisions”, Revue des deux mondes, n. ${ }^{\circ} 1$. París.

BERGER, Gastón. (1967), Etapes de la prospective, PUF.

DRUCKER, Peter. (2000), El management del siglo XXI. Barcelona: Edhasa.

DRUCKER, Peter. (2003), Gestión del conocimiento. Deusto, S.A., Ediciones.

GIL, Bolívar. (2005) "Inteligencia científica, tecnológica y regional". Manual del participante del Diplomado regional en gestión del conocimiento, Unisabana-Colciencia, Bogotá.

GODET, Michel; PROSPEKTIKER; FUTURIBLES. (1990), Problemas y Métodos de Prospectiva: Caja de Herramientas. UNESCO.

GODET, Michel. (1993), De la anticipación a la acción: Manual de prospectiva y estrategia., Marcombo Boixareu.

JOUVENEL, Hugues. (1993), "Sur la méthode prospective: un bref guide méthodologique", Futuribles, n. ${ }^{\circ} 179$.

MERA, Carlos. (2006). Prospectiva Estratégica. Bogotá. UNAD.

MERA, Carlos. (2009). Ponencia "La prospectiva estratégica herramienta para construir la esencia social comunitaria en las regiones al 2015". Foro Nacional de la Esencial Social Comunitaria. Bogotá. UNAD,

MINZBERG, Henry y QUINN, James. (2009), El Proceso Estratégico. Conceptos, Contextos y Casos. 2. ${ }^{a}$ edición. Pág. 5. Prentice Hall. México.

MOJICA, Francisco. (2005), La Construcción de Futuro. Bogotá: Editorial Universidad Externado de Colombia/Convenio Andrés Bello.

NONAKA, I. y TAKEUCHI, H. (1995), The Knowledge-Creating Company. How Japanese Companies Create the Dynamics of Innovation, Oxford University Press, New York/Oxford. 
NONAKA, I. Takeuchi, H. (1995), The concept of BA: building a foundation of knowledge creation. California Management Review. Vol.40. N. ${ }^{\circ} 3$.

NONAKA, I. Takeuchi, H. (1999), Organización creadora de conocimiento. México: Oxford University Press.

RUIZ, Alejandro. (1997), La Construcción del Futuro para la Gestión en la Complejidad, Ponencia presentada en el Rencontre. Dialectique du SAVOIR et du FAIRE.

SENGE, Peter. La quinta disciplina: como impulsar el aprendizaje en la organización inteligente. Barcelona. Gránica. 1999

SENGE, Peter. (2006), La quinta disciplina: El arte y la Práctica de la organización abierta al aprendizaje. Barcelona. Gránica. 


\section{ANEXO 1. \\ Árbol de Problemas}

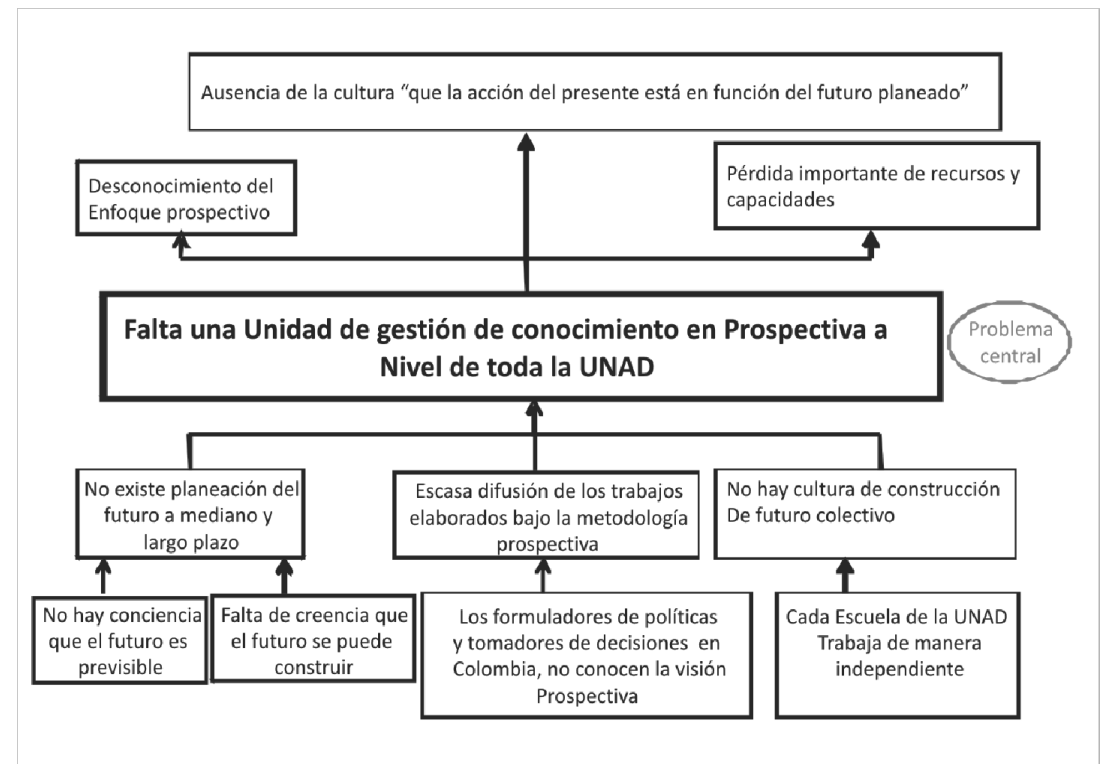

Fuente: Grupo de Investigaciones y Estudios Prospectivos y Estratégicos (GIEPE)

ANEXO 2.

\section{Planteamiento del Problema}

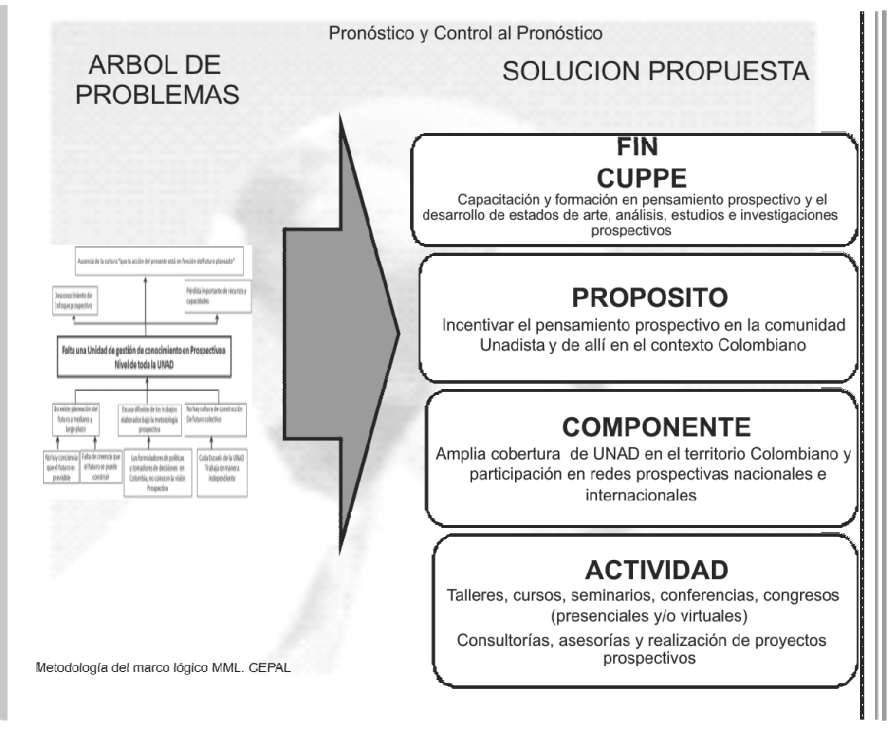




\section{ANEXO 3. \\ Benchmarking Centros Prospectivos del Mundo}

\begin{tabular}{|c|c|c|}
\hline Centros Prospectivos & Generalidades & Aplicaciones \\
\hline \multicolumn{3}{|c|}{ ARGENTINA } \\
\hline $\begin{array}{l}\text { Centro Latinoamericano de Globali- } \\
\text { zación y Prospectiva (CeLGyP) } \\
\text { www.millennium-project.org/ } \\
\text { millennium/Argentina } \\
\text { Presidente (in memoriam): } \\
\text { Dr. Horacio Godoy } \\
\text { Director: Miguel Ángel Gutiérrez } \\
\text { Secretario Ejecutivo: Adrián } \\
\text { Giraud } \\
\text { Coordinadora: Verónica Sandra } \\
\text { Peredo }\end{array}$ & $\begin{array}{l}\text { Desde } 2005 \text { es el Nodo } \\
\text { latinoamericano del Millennium } \\
\text { Project del American Council for } \\
\text { the United Nations (Universidad de } \\
\text { las Naciones Unidas). } \\
\text { Desarrolla programas de capacita- } \\
\text { ción avanzada e interactiva a través } \\
\text { de aulas digitales. } \\
\text { Realiza asesorías especiales en ma- } \\
\text { teria de reingeniería de empresas o } \\
\text { reformas institucionales gubernamen- } \\
\text { tales y nuevos planes educativos de } \\
\text { nivel universitario, en base al conoci- } \\
\text { miento del proceso de globalización. } \\
\text { Apoya a diversas instituciones, } \\
\text { como la Subsecretaría de Gestión } \\
\text { Pública, y promueve la creación de } \\
\text { cursos de Prospectiva y Centros de } \\
\text { Prospectiva en universidades. }\end{array}$ & $\begin{array}{l}\text { El desarrollo de los programas de } \\
\text { capacitación avanzada a través de } \\
\text { aulas virtuales. } \\
\text { El respaldo científico y académico } \\
\text { recibidodel Proyecto Milleniumpara } \\
\text { el desarrollo de sus actividades. } \\
\text { La promoción de cursos de prospectiva. }\end{array}$ \\
\hline $\begin{array}{l}\text { Centro de estudios prospectivos } \\
\text { de la Universidad de Cuyo } \\
\text { weblog.mendoza.edu.ar/universidad }\end{array}$ & $\begin{array}{l}\text { Se encuentra ubicado en la Facultad } \\
\text { de Ciencias Sociales. Actualmente } \\
\text { tiene acuerdos con diversas munici- } \\
\text { palidades, y coopera con los Insti- } \\
\text { tutos de energía, transporte, medio } \\
\text { ambiente, desarrollo social y de sa- } \\
\text { lud de la Universidad para adelantar } \\
\text { estudios prospectivos. }\end{array}$ & $\begin{array}{l}\text { Los acuerdos que hace con muni- } \\
\text { cipalidades y la cooperación con } \\
\text { estamentos de la Universidad para } \\
\text { adelantar estudios prospectivos. }\end{array}$ \\
\hline \multicolumn{3}{|c|}{ BOLIVIA } \\
\hline $\begin{array}{l}\text { Centro de profundización de la } \\
\text { democracia-CEPRODE } \\
\text { http://www.ceprode.org }\end{array}$ & $\begin{array}{l}\text { Organización no gubernamental sin } \\
\text { fines de lucro que actúa de manera } \\
\text { conjunta con Estado, Empresa y So- } \\
\text { ciedad Civil, para intervenir en los } \\
\text { asuntos públicos e incidir en las polí- } \\
\text { ticas nacionales de desarrollo orienta- } \\
\text { da a la producción de conocimientos. }\end{array}$ & $\begin{array}{l}\text { Busca que haya participación ciuda- } \\
\text { dana, sustentando valores importan- } \\
\text { tes para la sociedad. }\end{array}$ \\
\hline \multicolumn{3}{|c|}{ BRASIL } \\
\hline $\begin{array}{l}\text { Centro de Gestión de Estudios } \\
\text { Estratégicos-CGEE }\end{array}$ & $\begin{array}{l}\text { Asociación civil sin fines de lucro. } \\
\text { Promueve y realiza estudios de } \\
\text { alto nivel en ciencia y tecnología. } \\
\text { Promover y participar en estrategias } \\
\text { de evaluación y los impactos } \\
\text { económicos y sociales de las } \\
\text { políticas, programas y proyectos en } \\
\text { ciencia y tecnología. }\end{array}$ & $\begin{array}{l}\text { Es un difusor de información y } \\
\text { experiencias donde las empresas y } \\
\text { estudiantes pueden dar a conocer } \\
\text { sus proyectos. }\end{array}$ \\
\hline
\end{tabular}




\begin{tabular}{|c|c|c|}
\hline \multicolumn{3}{|c|}{ COLOMBIA } \\
\hline $\begin{array}{l}\text { Centro de Pensamiento estratégico } \\
\text { y Prospectiva } \\
\text { administracion.uexternado.edu.co/ } \\
\text { es/posgrado }\end{array}$ & $\begin{array}{l}\text { El Centro de Pensamiento Estratégico } \\
\text { y Prospectiva es un programa de apo- } \\
\text { yo a la Facultad de Administración de } \\
\text { Empresas que tiene como propósito } \\
\text { desarrollar una actitud mental que per- } \\
\text { mite incrementar la competitividad de } \\
\text { la organización iluminando las accio- } \\
\text { nes del presente con la luz del futuro. }\end{array}$ & $\begin{array}{l}\text { La prospectiva y la estrategia deno- } \\
\text { minadas "Ciencias de la esperanza" } \\
\text { Realiza asesorías, trabajos de con- } \\
\text { sultaría. }\end{array}$ \\
\hline $\begin{array}{l}\text { Centro Nacional de Productividad } \\
\text { Universidad del Valle. (Javier } \\
\text { Medina Vásquez) } \\
\text { cnp@cnp.org.co }\end{array}$ & $\begin{array}{l}\text { Creado para responder con rigor } \\
\text { metodológico hacia dónde y cómo } \\
\text { va a crecer Colombia los próximos } \\
\text { años, se enfoca en modelos de desa- } \\
\text { rrollo empresarial, social, basado en } \\
\text { la productividad, la competitividad y } \\
\text { la gestión enfocada a resultados me- } \\
\text { diante el Programa Colombiano de } \\
\text { Prospectiva Tecnológica Industrial. }\end{array}$ & $\begin{array}{l}\text { Realiza estudios en beneficio de la } \\
\text { población colombiana }\end{array}$ \\
\hline \multicolumn{3}{|c|}{ MÉXICO } \\
\hline $\begin{array}{l}\text { Prospectiva y Estrategia de la Fun- } \\
\text { dación Desarrollo Sustentable } \\
\text { www.lamolina.edu.pe/eventos/ } \\
\text { Vice_acad/Curso }\end{array}$ & $\begin{array}{l}\text { Creado para el estudiante de Cien- } \\
\text { cias Políticas, Ciencias de la Comu- } \\
\text { nicación, Sociología, Administración } \\
\text { Pública, Relaciones Internacionales y } \\
\text { carreras afines, aprenda los principa- } \\
\text { les postulados de la Prospectiva, como } \\
\text { disciplina de construcción de futuros, } \\
\text { así como dos herramientas computa- } \\
\text { rizadas que le ayudarán en el proceso } \\
\text { de hacer análisis prospectivo. }\end{array}$ & $\begin{array}{l}\text { Aplica la prospectiva para diferentes } \\
\text { disciplinas de carácter social. }\end{array}$ \\
\hline $\begin{array}{l}\text { Cipae - Centro Internacional de } \\
\text { Prospectiva Y Altos Estudios } \\
\text { www. cipae. ed } u . m x / \text { to o ls } / \\
\text { CONVOCferia2.pdf }\end{array}$ & $\begin{array}{l}\text { Es una institución privada que, me- } \\
\text { diante la producción del saber, desarro- } \\
\text { lla y aplica modelos educativos, meto- } \\
\text { dologías y estrategias para promover el } \\
\text { desarrollo humano y brindar asesoría a } \\
\text { entidades públicas y privadas. }\end{array}$ & $\begin{array}{l}\text { Esta en diferentes partes del mundo } \\
\text { (Argentina, Australia, Bélgica) }\end{array}$ \\
\hline \multicolumn{3}{|c|}{ ESPAÑA } \\
\hline $\begin{array}{l}\text { Centro Andaluz de prospectiva } \\
\text { C.A.N.P } \\
\text { http://www.centrodeestudiosandaluces.es }\end{array}$ & $\begin{array}{l}\text { Entidad de carácter científico y cultural, } \\
\text { sin ánimo de lucro, que establece el } \\
\text { fomento de la investigación científica, } \\
\text { la generación de conocimiento sobre la } \\
\text { realidad social, económica y cultural de } \\
\text { Andalucía y la difusión de sus resultados } \\
\text { en beneficio de toda la sociedad. } \\
\text { Adscrito a la Consejería de Presiden- } \\
\text { cia. Se rige por un Patronato, que ejer- } \\
\text { ce como órgano de representación, go- } \\
\text { bierno y administración, un Presidente } \\
\text { y un Director gerente. Cuenta, además, } \\
\text { con un Comité Científico que actúa } \\
\text { como órgano asesor y que está com- } \\
\text { puesto por reconocidas personalidades } \\
\text { del mundo de la ciencia y la cultura. }\end{array}$ & $\begin{array}{l}\text { La generación del amplio programa } \\
\text { de actividades anuales abiertas a la } \\
\text { comunidad científica y a la sociedad } \\
\text { en general. }\end{array}$ \\
\hline
\end{tabular}




\begin{tabular}{|c|c|c|}
\hline $\begin{array}{l}\text { Instituto Español de Prospectiva } \\
\text { en la Educación Superior (IEPES) } \\
\text { www.educaweb.com }\end{array}$ & $\begin{array}{l}\text { Grupo editorial y formativo vincula- } \\
\text { do a distintas universidades españo- } \\
\text { las, con la necesidad de establecer } \\
\text { una clara relación con los princi- } \\
\text { pios, los objetivos, el desarrollo } \\
\text { metodológico y los resultados más } \\
\text { aplicados de la Convergencia Euro- } \\
\text { pea en cada Universidad española. } \\
\text { ElIEPES trata de establecer un puente } \\
\text { entre los diseñadores de las políticas } \\
\text { de la Educación Superior en la Unión } \\
\text { Europea y las Universidades públicas } \\
\text { y privadas buscando intensificar } \\
\text { la relación entre la industria, la } \\
\text { empresa y la Universidad, por medio } \\
\text { de la Actualización del diseño de los } \\
\text { grados, postgrados y doctorados que } \\
\text { se desarrollan en Europa. }\end{array}$ & $\begin{array}{l}\text { Es un centro del sector privado. } \\
\text { Busca intensificar la relación entre } \\
\text { industria-empresa-universidades. }\end{array}$ \\
\hline $\begin{array}{l}\begin{array}{l}\text { Centro De estudios de } \\
\text { Prospectiva }\end{array} \\
\text { www.nebrija.com/prospectiva.../ }\end{array}$ & $\begin{array}{l}\text { Creadores del proyecto MADRID } \\
\text { PROSPECTIVA } 2015 \text { que sintetiza } \\
\text { un conjunto de análisis de tenden- } \\
\text { cias -demográficas, económicas, } \\
\text { tecnológicas....- que lleven a definir } \\
\text { una serie de "Imágenes de Madrid". }\end{array}$ & $\begin{array}{l}\text { Realizan estudios prospectivos a } \\
\text { partir de métodos estadísticos. }\end{array}$ \\
\hline \multicolumn{3}{|c|}{ CHINA } \\
\hline \multirow[t]{2}{*}{$\begin{array}{lll}\text { Centro } & \text { Internacional } & \text { de } \\
\text { Prospectiva } & \text { y Altos Estudios }\end{array}$} & $\begin{array}{l}\text { Participó conjuntamente con la } \\
\text { UNESCO y el Ministerio de Educa- } \\
\text { ción Chino en el desarrollo de tec- } \\
\text { nología educativa para la formación } \\
\text { de Ingenieros en electrónica. (casa } \\
\text { de los niños, teatro guiñol, entre } \\
\text { muchos otros) El CIPAE participó } \\
\text { con el Ministerio de Educación de } \\
\text { Japón en la reunión sobre tecnolo- } \\
\text { gías educativas realizada en Tokio. } \\
\text { CIPAE participó con propuestas } \\
\text { novedosas en diversos aspectos para } \\
\text { el desarrollo de dichas tecnologías. }\end{array}$ & $\begin{array}{l}\text { Sus estudios son realizados a partir } \\
\text { del desarrollo de tecnología en } \\
\text { educación para la formación de } \\
\text { futuros ingenieros en electrónica. }\end{array}$ \\
\hline & TAHILANDIA & \\
\hline $\begin{array}{l}\text { Centro Internacional de } \\
\text { Prospectiva y Altos Estudios. } \\
\text { www.cipae.edu.mx/ }\end{array}$ & $\begin{array}{l}\text { El Centro Internacional de Prospecti- } \\
\text { va y Altos Estudios participó conjun- } \\
\text { tamente con la UNESCO y el Minis- } \\
\text { terio de Educación de Costa de Marfil } \\
\text { Debido a los desastres provocados } \\
\text { por la guerra civil en la República de } \\
\text { Mozambique en el sistema escolar, el } \\
\text { país requirió reformular con urgencia, } \\
\text { políticas educativas y métodos de im- } \\
\text { plantación de nuevos procedimientos } \\
\text { de atención en el sistema escolar. }\end{array}$ & $\begin{array}{l}\text { Estudios prospectivos para refor- } \\
\text { mular políticas educativas en el } \\
\text { sistema escolar }\end{array}$ \\
\hline
\end{tabular}

Fuente: Grupo de Investigaciones y Estudios Prospectivos y Estratégicos (GIEPE) 


\author{
ANEXO 4. \\ Método Delphi - Expertos
}

UNIVERSIDAD NACIONAL ABIERTA Y A DISTANCIA -UNAD

GRUPO DE INVESTIGACIONES Y ESTUDIOS PROSPECTIVOS Y

ESTRATÉGICOS -GIEPE

\title{
ESTUDIO DE FACTIBILIDAD PARA LA CREACIÓN DEL CENTRO \\ UNADISTA DE PENSAMIENTO PROSPECTIVO Y ESTRATÉGICO -CUPPE ENCUESTA DELPHI A EXPERTOS
}

\section{Datos del encuestado}

Nombre y Apellido:

Fecha de nacimiento:

Sexo:

Correo electrónico:

País / Ciudad de ubicación:

Teléfono:

El Grupo de Investigaciones y Estudios Prospectivos y Estratégicos GIEPE de la Universidad Nacional Abierta y a Distancia UNAD en el marco del Estudio de factibilidad para la creación del Centro Unadista de Pensamiento Prospectivo y Estratégico cuppe, se complace en invitarlo en su calidad de experto a diligenciar la siguiente encuesta.

Dicha encuesta consta de un cuestionario con cuatro áreas temáticas relativas a la creación del Centro Unadista de Pensamiento Prospectivo y Estratégico (CuPPE), cada una incluye preguntas a las que deberán asignarle una calificación de 1 a 5, teniendo en cuenta que 1 es la calificación más baja y 5 la más alta.

Agradecemos su valiosa participación en este ejercicio, seguros de que será de gran utilidad para el desarrollo futuro del CUPPE. Sus importantes aportes en el diligenciamiento de esta encuesta, tendrán el reconocimiento debido en las publicaciones resultantes de éste estudio, que gustosos les enviaremos en agradecimiento una vez que lo hayamos concluido.

\begin{tabular}{|c|c|c|c|c|c|c|}
\hline Debe seleccionar una opción & 1 & 2 & 3 & 4 & 5 & NS \\
\hline $\begin{array}{l}\text { 1. ¿En qué grado comparte la siguiente definición: "lo que hace el futuro es } \\
\text { nuestra capacidad de actuar?" }\end{array}$ & & & & & & \\
\hline $\begin{array}{l}\text { 2. ¿Cree usted que la prospectiva es una herramienta metodológica que } \\
\text { contribuye en la solución de problemas complejos? }\end{array}$ & & & & & & \\
\hline $\begin{array}{l}\text { 3. ¿Qué tanto cree usted que la prospectiva influye en la transformación } \\
\text { económica y social? }\end{array}$ & & & & & & \\
\hline $\begin{array}{l}\text { 4. ¿La prospectiva aporta a la construcción de futuros que mejoren la calidad } \\
\text { de vida? }\end{array}$ & & & & & & \\
\hline
\end{tabular}


Dentro de la misión de la Universidad Nacional Abierta y a Distancia - UNAD está contribuir a la educación para todos, mediante la investigación, la acción pedagógica, la proyección social y las innovaciones metodológicas y didácticas aportando al desarrollo humano sostenible de las comunidades locales y globales, con calidad, eficiencia y equidad social.

\begin{tabular}{|c|c|c|c|c|c|c|}
\hline Debe seleccionar una opción & 1 & 2 & 3 & 4 & 5 & NS \\
\hline $\begin{array}{l}\text { 5. Cree usted que la creación del CUPPE contribuye a la formación del } \\
\text { pensamiento proactivo en la Comunidad Unadista, convirtiéndola en una } \\
\text { comunidad que genere sociedades futuras sostenibles capaces de reaccionar } \\
\text { ante los constantes cambios de este mundo cada vez más globalizado? }\end{array}$ & & & & & & \\
\hline $\begin{array}{l}\text { 6. ¿Considera que la creación del CUPPE sustenta el rol que juega la UNAD, como } \\
\text { un espacio social, científico, tecnológico y cultural, orientado a producir y } \\
\text { transmitir conocimiento con calidad y excelencia académica, aplicado a la } \\
\text { solución de los grandes problemas nacionales y regionales, el pensamiento } \\
\text { y la creatividad? }\end{array}$ & & & & & & \\
\hline $\begin{array}{l}\text { 7. ¿Considera usted que la organización que comprenda el proceso de } \\
\text { evolución del entorno y que pueda conocer su plan de acción antes que otras } \\
\text { organizaciones, se beneficiará de una ventaja competitiva, posibilitándole } \\
\text { adoptar con anticipación una mejor estrategia? }\end{array}$ & & & & & & \\
\hline
\end{tabular}

Al implementarse el CUPPE se quiere formar académicamente para la investigación utilizando la metodología prospectiva, además de prestar servicios de formación, formulación de proyectos de investigación, asesorías, consultorías, difusión de experiencias investigativas, planeación y desarrollo de proyectos de construcción de futuros y creación de redes de apoyo a la investigación.

\begin{tabular}{|c|c|c|c|c|c|c|}
\hline Debe seleccionar una opción & 1 & 2 & 3 & 4 & 5 & NS \\
\hline $\begin{array}{l}\text { 8. ¿Considera que el mejor medio de comunicación para dar a conocer el } \\
\text { CUPPE y lograr interacción tanto con la comunidad Unadista como con el } \\
\text { medio exterior es la página web? }\end{array}$ & & & & & & \\
\hline $\begin{array}{l}\text { 9. ¿Cree usted que el mejor medio para difundir sus servicios es el espacio } \\
\text { virtual? }\end{array}$ & & & & & & \\
\hline $\begin{array}{l}\text { 10. ¿En qué grado considera que las Tecnologías de Información y Comunicación } \\
\text { contribuyen a una mayor integración y participación en la construcción de } \\
\text { escenarios prospectivos? }\end{array}$ & & & & & & \\
\hline
\end{tabular}

Siendo la UNAD un contexto sociocultural de actuación nacional e internacional en el que se promueve y circula la gestión del conocimiento, mediante una estructura compuesta por nodos (componente estructural considerado como núcleo de interrelación entre varias redes con capacidad tecnológica académica y administrativa) que une diferentes Centros de Educación a Distancia CEAD. Centros Regionales de Educación Superior - CERES y Centros Comunitarios de Atención Virtual - CCAV. ¿En qué área de la Prospectiva cree usted que debe especializarse el CUPPE? 


\begin{tabular}{|l|l|l|l|l|l|l|}
\hline Debe seleccionar una opción & $\mathbf{1}$ & $\mathbf{2}$ & $\mathbf{3}$ & $\mathbf{4}$ & $\mathbf{5}$ & NS \\
\hline Prospectiva territorial & & & & & & \\
\hline Prospectiva tecnológica & & & & & & \\
\hline Prospectiva estratégica & & & & & & \\
\hline Prospectiva organizacional & & & & & & \\
\hline Otras & & & & & & \\
\hline
\end{tabular}

Si su respuesta es Otras ¿Cuáles?

\section{ANEXO 5. \\ Plataforma Prospectiva de la UNAD}

Plataforma Prospectiva de la UNAD

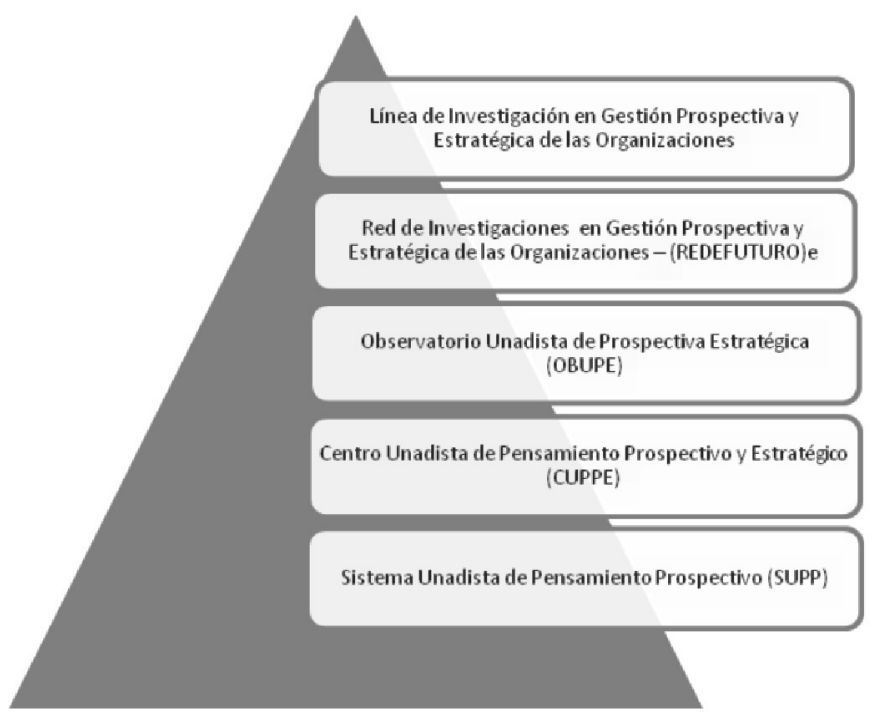

Fuente: Grupo de Investigaciones y Estudios Prospectivos y Estratégico - GIEPE

En donde el Sistema Unadista de Pensamiento Prospectivo y Estratégico - suPp, es el sistema que organiza las acciones de la Prospectiva estratégica en la Universidad y garantiza que los diversos subsistemas académicos, de gestión se correlacionen y interactúen de forma adecuada, el suPP es responsable de garantizar las alianzas internas y externas de la UNAD 
con otras instituciones y otros sectores y que garantice un mayor impacto social, económico y ambiental.

El Centro Unadista de Pensamiento Prospectivo cuppe, es el núcleo gestor de conocimiento especializado en prospectiva estratégica que toma la figura de Unidad empresarial que apoya a la Escuela de Ciencias Administrativas, Contables, Económicas y de Negocios de la Universidad Nacional Abierta y a Distancia, por medio de estudios, análisis, planes de futuro que le den solución a las problemáticas Locales, Regionales y Nacionales.

El Observatorio Unadista de Prospectiva Estratégica, es una herramienta muy poderosa en el tema de la TIC que se encuentra alojado en el CUPPE y tiene por objetivo ser el sistema de información, en permanente actualización, que apoya la plataforma prospectiva de la UNAD en temas relacionados con la prospectiva regional, tecnológica, política, organización y en gestión del conocimiento por medio de las nuevas tecnologías de la información y comunicación, con el fin de evaluar cómo evolucionan las tendencias y Megatendencias mundiales.

La Red de Prospectiva estratégica busca integrar a los grupos, semilleros, investigadores, estudiantes, egresados y expertos nacionales e internacionales, que trabajen en esta línea, brindándoles un espacio para la construcción de un mejor futuro para las próximas generaciones. La vinculación de la investigación a la actividad de formación se puede hacer con mayor sentido si se organizan los Grupos como redes académicas de conocimiento, en las cuales los contenidos producidos son objeto de contextualización y reconstrucción por los actores participantes, e integran los resultados de investigación. Así cada desarrollo de un Grupo generaría nuevas producciones del cuerpo académico.

La Línea de Investigación es la ordenadora de la actividad de investigación, con un eje temático y común de problemas que facilita la integración y continuidad de los esfuerzos en forma ordenada y sistemática, con el propósito de abordar en forma cooperada e interdisciplinaria un campo de conocimiento alrededor de la cual se articulan investigadores, proyectos, problemas, metodologías y actividades de investigación que hacen posible la producción intelectual en un campo del saber. 


\section{ANEXO 6. \\ Resultados esperados \\ Generación de nuevo conocimiento}

\begin{tabular}{|l|l|ll|}
\hline \multicolumn{1}{|c|}{ Resultado/Producto esperado } & \multicolumn{1}{|c|}{ Indicador } & \multicolumn{1}{|c|}{ Beneficiario } \\
\hline $\begin{array}{l}\text { Creación e implementación del } \\
\text { Centro de Pensamiento Prospectivo }\end{array}$ & $\begin{array}{l}\text { Norma de Creación del CUPPE en } \\
\text { la UnAD }\end{array}$ & $\begin{array}{l}\text { Sociedad, Empresas, Estado, } \\
\text { Comunidad Unadista. }\end{array}$ \\
\hline $\begin{array}{l}\text { Crear e implementar el Observatorio } \\
\text { de Prospectiva Estratégica } \\
\text { (OBUPE), como el centro de } \\
\text { sistemas de información. }\end{array}$ & $\begin{array}{l}\text { Norma de Creación del OBUPPE en } \\
\text { la UnAD }\end{array}$ & $\begin{array}{l}\text { Sociedad, empresa, estado, } \\
\text { Comunidad Unadista. }\end{array}$ \\
\hline $\begin{array}{l}\text { Adquisición, diseño y aplicación de } \\
\text { nuevo Software. Como herramienta } \\
\text { para desarrollar investigaciones de } \\
\text { Prospectiva (FUTURO) }\end{array}$ & $\begin{array}{l}\text { Número de Proyectos utilizando } \\
\text { software Prospectivo. }\end{array}$ & $\begin{array}{l}\text { Sociedad, Empresas, Estado y } \\
\text { Comunidad Unadista. }\end{array}$ \\
\hline $\begin{array}{l}\text { Diseño de la metodología } \\
\text { prospectiva para proyectos de } \\
\text { investigación }\end{array}$ & $\begin{array}{l}\text { Proyectos de Investigación } \\
\text { presentados bajo la metodología } \\
\text { diseñada. }\end{array}$ & $\begin{array}{ll}\text { Sociedad, empresa, estado y } \\
\text { Comunidad Unadista. }\end{array}$ \\
\hline
\end{tabular}

\section{Fortalecimiento de la Comunidad cientifica}

\begin{tabular}{|l|l|l|}
\hline \multicolumn{1}{|c|}{ Resultado/Producto esperado } & \multicolumn{1}{|c|}{ Indicador } & \multicolumn{1}{c|}{ Beneficiario } \\
\hline $\begin{array}{l}\text { Creación de la red de investigación } \\
\text { en Gestión del conocimiento } \\
\text { prospectivo y estratégico. }\end{array}$ & $\begin{array}{l}\text { Número de grupos de investigación } \\
\text { y semilleros articulados a la Red }\end{array}$ & $\begin{array}{l}\text { Comunidad Unadista, Redes } \\
\text { Nacionales e Internacionales }\end{array}$ \\
\hline $\begin{array}{l}\text { Pertenecer a una red de prospectiva } \\
\text { o estudios de futuro a nivel nacional } \\
\text { e internacional }\end{array}$ & $\begin{array}{l}\text { Número de Redes a las que pertenece } \\
\text { el CUPPE. }\end{array}$ & $\begin{array}{l}\text { Investigadores, semilleros, grupos y } \\
\text { redes de investigación. }\end{array}$ \\
\hline $\begin{array}{l}\text { Trabajos de grado relacionado con } \\
\text { las temáticas desarrolladas por el } \\
\text { CUPPE }\end{array}$ & $\begin{array}{l}4 \text { Trabajos de grados presentados y y } \\
\text { avalados por el GIEPE y el CUPPE. } \\
\text { Como Opción de Grado. }\end{array}$ & $\begin{array}{l}\text { Investigadores, Auxiliares de } \\
\text { Investigación, Semilleros, Siunad, } \\
\text { Comunidad Unadista. }\end{array}$ \\
\hline $\begin{array}{l}\text { Convenios y alianzas con Centros, } \\
\text { instituciones, asociaciones, } \\
\text { federaciones relacionadas con los } \\
\text { estudios de futuro. }\end{array}$ & $\begin{array}{l}2 \text { alianzas o convenios de } \\
\text { cooperación o colaboración Unad } \\
-\mathrm{X}\end{array}$ & $\begin{array}{l}\text { Empresas, Sociedad, Estado, } \\
\text { Comunidad Unadista. }\end{array}$ \\
\hline
\end{tabular}

\section{Apropiación Social del Conocimiento}

\begin{tabular}{|l|l|l|}
\hline \multicolumn{1}{|c|}{ Resultado/Producto esperado } & \multicolumn{1}{|c|}{ Indicador } & \multicolumn{1}{c|}{ Beneficiario } \\
\hline $\begin{array}{l}\text { Consolidación y fortalecimiento } \\
\text { del curso de Profundización en } \\
\text { Prospectiva Estratégica. }\end{array}$ & $\begin{array}{l}\text { Realizar en el 2010,6 cursos a nivel } \\
\text { nacional (En los CEAD). }\end{array}$ & $\begin{array}{l}\text { Sociedad, empresa, estado, } \\
\text { Comunidad Unadista. }\end{array}$ \\
\hline $\begin{array}{l}\text { Consolidación de Prospecta } \\
\text { Colombia - Congreso internacional } \\
\text { de prospectiva estratégica. }\end{array}$ & $\begin{array}{l}\text { Realizar un Congreso Internacional } \\
\text { anualmente. }\end{array}$ & $\begin{array}{l}\text { Sociedad, empresa, estado, } \\
\text { Comunidad Unadista, Comunidad } \\
\text { Académica Internacional }\end{array}$ \\
\hline
\end{tabular}




\begin{tabular}{|l|l|l|}
\hline $\begin{array}{l}\text { Diseño y elaboración de guías y } \\
\text { módulos de prospectiva estratégica }\end{array}$ & $\begin{array}{l}\text { Una guía y un módulo del curso de } \\
\text { prospectiva. }\end{array}$ & $\begin{array}{l}\text { Sociedad, empresa, } \\
\text { Comunidad Unadista. }\end{array}$ \\
\hline $\begin{array}{l}\text { Elaboración de artículos de } \\
\text { investigación prospectiva }\end{array}$ & 2 artículos por año. & $\begin{array}{l}\text { Sociedad, empresa, estado, } \\
\text { Comunidad Unadista. }\end{array}$ \\
\hline $\begin{array}{l}\text { Presentar ponencias a nivel nacional } \\
\text { e internacional }\end{array}$ & $\begin{array}{l}\text { 2ponencias nacionales y 2 ponencias } \\
\text { internacionales por año }\end{array}$ & $\begin{array}{l}\text { Sociedad, empresa, estado, } \\
\text { Comunidad Unadista. }\end{array}$ \\
\hline $\begin{array}{l}\text { Diseño de la propuesta Diplomado } \\
\text { en prospectiva regional. }\end{array}$ & $\begin{array}{l}\text { Una propuesta para presentar en el } \\
\text { primer trimestre del 2010 }\end{array}$ & $\begin{array}{l}\text { Sociedad, empresa, estado, } \\
\text { Comunidad Unadista. }\end{array}$ \\
\hline $\begin{array}{l}\text { Diseño de la propuesta para } \\
\text { la Maestría en Prospectiva } \\
\text { Organizacional. }\end{array}$ & $\begin{array}{l}\text { Propuesta para presentar en el elando trimestre del 2010. } \\
\text { segundo, }\end{array}$ & $\begin{array}{l}\text { Sociedad, estado, } \\
\text { Comunidad Unadista. }\end{array}$ \\
\hline
\end{tabular}

\section{Impactos esperados}

\begin{tabular}{|c|c|c|c|}
\hline Impacto esperado & $\begin{array}{c}\text { Plazo (años) después de } \\
\text { finalizado el proyecto: } \\
\text { corto }(1-4) \\
\text { mediano }(5-9) \\
\text { largo (10 o más) }\end{array}$ & $\begin{array}{l}\text { Indicador } \\
\text { verificable }\end{array}$ & Supuestos* \\
\hline $\begin{array}{l}\text { Reconocimiento del } \\
\text { CUPPE a nivel nacional e } \\
\text { internacional. }\end{array}$ & $\begin{array}{l}\text { Lograr el reconocimiento } \\
\text { En el } 2011 \text { a nivel institucio- } \\
\text { nal. } \\
\text { En el } 2015 \text { a nivel nacional. } \\
\text { En el } 2019 \text { a nivel internacio- } \\
\text { nal. }\end{array}$ & $\begin{array}{l}\text { Proyectos realizados } \\
\text { Redes a las que per- } \\
\text { tenece Congresos } \\
\text { realizados }\end{array}$ & $\begin{array}{l}\text { Apoyo financiero por } \\
\text { parte de la universidad }\end{array}$ \\
\hline $\begin{array}{l}\text { Crear } 8 \text { Subcentros de } \\
\text { Gestión de Conocimiento } \\
\text { Especializado en Prospec- } \\
\text { tiva Estratégica en cada } \\
\text { uno de los Nodos de la } \\
\text { UNAD - Articulados al CU- } \\
\text { PPE en Red. }\end{array}$ & $\begin{array}{l}\text { Crear } 8 \text { Centros en los Nodos } \\
\text { de la UNAD } \\
\text { En el } 2011 \text { Crear } 2 \text { Centros } \\
\text { En el } 2015 \text { Crear } 3 \text { Centros } \\
\text { En el } 2019 \text { Crear } 3 \text { Centros }\end{array}$ & $\begin{array}{l}8 \text { Subcentros de Ges- } \\
\text { tión de Conocimien- } \\
\text { to Especializados en } \\
\text { Prospectiva Estraté- } \\
\text { gica }\end{array}$ & $\begin{array}{l}\text { Con el apoyo Económico } \\
\text { de la UNAD }\end{array}$ \\
\hline $\begin{array}{l}\text { Brindar capacitación y } \\
\text { asesorías en prospectiva a } \\
\text { la comunidad en general. }\end{array}$ & $\begin{array}{l}\text { En el } 2011 \text { ser el líder en } \\
\text { asesorías y capacitaciones en } \\
\text { lo que a prospectiva se refiere. }\end{array}$ & $\begin{array}{l}\text { En el } 2011 \text { ser el } \\
\text { líder en asesorías y } \\
\text { capacitaciones en lo } \\
\text { que a prospectiva se } \\
\text { refiere. }\end{array}$ & $\begin{array}{l}\text { Capacitar a los docen- } \\
\text { tes y tutores por medio } \\
\text { de cursos y talleres con } \\
\text { prospectivistas interna- } \\
\text { cionales. }\end{array}$ \\
\hline $\begin{array}{l}\text { Fomentar la actitud y el } \\
\text { pensamiento prospectivo en } \\
\text { la comunidad en general. }\end{array}$ & $\begin{array}{l}\text { En el } 2011 \text { que al menos el } \\
30 \% \text { de la comunidad Unadis- } \\
\text { ta reconozca y visualice la im- } \\
\text { portancia de la prospectiva. }\end{array}$ & $\begin{array}{l}\text { Número de personas } \\
\text { pensando prospectiva- } \\
\text { mente (en el futuro) }\end{array}$ & $\begin{array}{l}\text { Tener mayor cobertura a } \\
\text { nivel nacional en los CEAD } \\
\text { conformando semilleros } \\
\text { de investigación. }\end{array}$ \\
\hline
\end{tabular}

